\title{
Measurement of deforming mode of lattice truss structures under impact loading
}

\author{
J. Liu ${ }^{1}$, S. Pattofatto ${ }^{2}$, and H. Zhao ${ }^{1, a}$ \\ ${ }^{1}$ Laboratoire de Mécanique et Technologie de Cachan, 61 avenue du Président Wilson, 94230 Cachan, \\ France \\ ${ }^{2}$ SNECMA-Site de Villaroche, Rond-Point René Ravaud, 77550 Moissy-Cramayel, France
}

\begin{abstract}
Lattice truss structures, which are used as a core material in sandwich panels, were widely investigated experimentally and theoretically. However, explanation of the deforming mechanism using reliable experimental results is almost rarely reported, particularly for the dynamic deforming mechanism. The present work aimed at the measurement of the deforming mode of lattice truss structures. Indeed, quasi-static and Split Hopkinson Pressure Bar (SHPB) tests have been performed on the tetrahedral truss cores structures made of Aluminum 3003-O. Global values such as crushing forces and displacements between the loading platens are obtained. However, in order to understand the deforming mechanism and to explain the observed impact strength enhancement observed in the experiments, images of the truss core element during the tests are recorded. A method based on the edge detection algorithm is developed and applied to these images. The deforming profiles of one beam are extracted and it allows for calculating the length of beam. It is found that these lengths diminish to a critical value (due to compression) and remain constant afterwards (because of significant bending). The comparison between quasi-static and impact tests shows that the beam were much more compressed under impact loading, which could be understood as the lateral inertia effect in dynamic bucking. Therefore, the impact strength enhancement of tetrahedral truss core sandwich panel can be explained by the delayed buckling of beam under impact (more compression reached), together with the strain hardening of base material.
\end{abstract}

\section{Introduction}

Cellular materials (such as honeycomb, foam and hollow sphere agglomerate) combining both lightweight and high energy absorbing capacity are widely used in various industrial areas, such as automobiles, naval vehicles, aircrafts [1]. Sandwich panel containing honeycomb cores is one kind of superior material comparing with stochastic foams, like CYMAT foams and Alporas foams. However, most commercial honeycomb core panels are difficult to fabricate into complex curved shapes because of induced anticlastic curvature, and they also trap moisture leading to internal corrosion and facesheet debonding due to their close-cell structure (indicated by Sypeck 2005 [2]). With the development of manufacturing techniques, lattice truss cores structures like triangular or pyramidal truss core topologies attracted a lots of attentions of researchers in last decade. As an open cell periodic structure, lattice truss core structure can exploit to multifunctional applications, for example, heat exchange media [3]. It can also decrease the relative density of the structures to several percents. Therefore, lattice truss core structure is supposed to be a promising substitute.

Lots of theoretical prediction works on the mechanical responses of lattice truss cores structures were done in the last decade $[4,5]$. The manufacture methods and mechanical behaviors were comprehensively reviewed by Wadley [6] in 2006; while Zok et al. [7] performed the transverse compression, in-plane shear and stretch on 304 stainless pyramidal truss cores, and then established an orthotropic constitutive law for the core. All the works above are concentrated in the quasi-static responses of

a e-mail: zhao@lmt.ens-cachan.fr these materials. In dynamic aspects, the mitigation of blast wave [8-11] and ballistic perforation [12] were conducted theoretically, experimentally and even numerically. They revealed the dynamic response of the whole sandwich panel. However, to understand thoroughly the dynamic behavior of lattice truss core structures and establish the constitutive model of these structures, the response of the single core under impact is obligatory.

Lee et al. [13] executed a comprehensive research on pyramidal truss core under various strain rate from $10^{-3} \mathrm{~s}^{-1}$ (quasi-static) to $10^{2}-10^{3} \mathrm{~s}^{-1}$ (Hopkinson bar), and up to $10^{4} \mathrm{~s}^{-1}$ (gas gun). However, they also limited only to the phenomenal behavior of the structure (the overall response under out-of-plane compression). In this paper, quasi-static and dynamic compressive experimental tests on the tetrahedral truss core made from rate insensitive Al3003-O are performed. With the digital images analysis, the failure mechanism of lattice truss core structure is highlighted.

\section{Material and method}

\subsection{Manufacture of tetrahedral truss core material}

The specimen is composed of two face sheets and the cores sandwiched between these sheets. The core comprises three struts or beams which cross together as a regular tetrahedron (as shown in figure 1) with the beams $15.31 \mathrm{~mm}$ in length, $2 \mathrm{~mm}$ in width and $1 \mathrm{~mm}$ in thickness. The height of the core is then $12.5 \mathrm{~mm}$ while the thickness of the face sheets is $1 \mathrm{~mm}$. The relative density of the core is thus $3.6 \%$. 


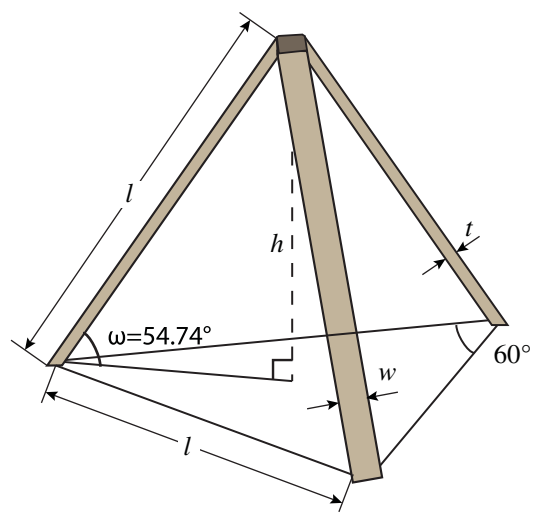

Fig. 1. The tetrahedral truss core specimen.

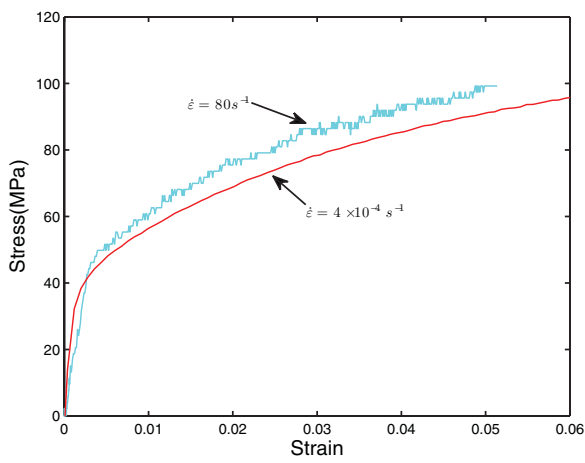

Fig. 2. Measured quasi-static and dynamic uniaxial response of aluminum 3003-O.

The specimens both the face sheets and the cores are manufactured from aluminum 3003-O with density $2730 \mathrm{Kg} / \mathrm{m}^{3}$. The quasi-static and dynamic uniaxial tensile experiments of the base material have been performed on Instron 5882 testing system and $10 \mathrm{~mm}$ diameter Hopkinson tensile bars separately.

In the uniaxial tensile testing, especially the dynamic tensile tests, the specimen can't be clamped absolutely firmly. During loading, the grips will always slip over the specimen surface where it's clamped. As a result, the strains measured with the traditional method (strain gages in Hopkinson bar and directly given by testing system) are not accurate. To overcome this drawback, a digital image correlation method (Correli ${ }^{Q 4}$ [14] programed by LMT-Cachan) is applied to the images shot during testing to measure the strain in the specimen. Assuming the deforming state is homogeneous, and then the average strain is contributed to the stress-strain curve.

As shown in figure 2, the strain rate effect of aluminum is not obvious in the range of 6 orders (from $10^{-4} \mathrm{~s}^{-1}$ to $\left.10^{2} \mathrm{~s}^{-1}\right)$. Meanwhile, the stress-strain curve of aluminum 3003-O manifests strong strain hardening capacity. When the strain increases to $3 \%$ the strength increases to two times of the yield strength.

\subsection{Experimental set-up}

The quasi-static compression tests are performed on MTS 810 material testing system, which is a well-controlled technique. It provides that the suitable device is used for the force measurement when the very weak foamlike material are tested. However, experimental techniques differ when the strain rate is important. Rapid hydraulic testing machine $[15,16]$ or falling weight arrangements $[17,18]$ are techniques often reported in the literature. Even those techniques can provide a suitable mechanical loading over large range of strain rates, the quality of the measuring approaches associated with those techniques decreases with the increase of strain rates. This is because they both assume an instantaneous equilibrium not only in the loading frame but also in the measuring device. This assumption neglects any wave propagation effect, which is very important in dynamic experiments. As a result, those measuring techniques begin to lose accuracy at medium strain rates $(\dot{\varepsilon}>50 / \mathrm{s})$ and the special short loads cells have to be used. For the high strain rates $(\dot{\varepsilon}>200 /$ s), it is very difficult to obtain reliable results using those devices.

The Split Hopkinson Pressure Bar (SHPB) is a widely used experimental technique to study the constitutive relationship of materials in high strain rates $[19,20]$. A typical SHPB is made of long input and output pressure bars with a short specimen sandwiched between them. The impact between the projectile and the input bar generates a compressive longitudinal incident pulse $\varepsilon_{i}(t)$ in the input bar. Once this incident pulse reaches the specimen-bar interface, one part is reflected as reflected pulse $\varepsilon_{r}(t)$ in the input bar and the other part transmitted as transmitted pulse $\varepsilon_{t}(t)$ in the output bar. With the gauges glued on the input bar and output bar, one can record these three pulses, and then forces and velocities at both interface of the specimen are given by the following:

$$
\begin{gathered}
F_{\text {input }}=S_{B} E\left(\varepsilon_{i}(t)+\varepsilon_{r}(t)\right), \\
F_{\text {output }}=S_{B} E\left(\varepsilon_{t}(t)\right) \\
\text { and } \\
V_{\text {input }}=C_{0}\left(\varepsilon_{i}(t)-\varepsilon_{r}(t)\right), \\
V_{\text {output }}=C_{0} \varepsilon_{t}(t)
\end{gathered}
$$

where $S_{B}, E$ and $C_{0}$ denote cross sectional area, Young's modulus and wave speed in the bar.

On the use of Hopkinson bar, one important assumption is the homogeneity of the specimen during loading. However, in most cases of soft materials with intense localized deformation, for example, the lattice truss cores structure presented in this paper, the homogeneous assumption is not valid. Nevertheless, we introduce the output force as a function of the crush of the specimen $\Delta(t)$ to give an overall idea of the behavior of the material tested, where the crush of the specimen is defined as:

$$
\Delta(t)=\int_{0}^{t}\left(V_{\text {output }}(\tau)-V_{\text {input }}(\tau)\right) d \tau
$$

In order to improve the accuracy of measuring, the soft, large diameter nylon bar should be used to hold the big size $(51.5 \mathrm{~mm}$ in edge), low strength specimen in our experiments. However, the previous reasoning can't describe the wave dispersion effect in a large diameter visco-elastic bar which can introduce some error in the necessary virtual shift from the measuring point of strain to the bar-specimen interface in time and space. The 
correction of this dispersion effect on the basis of a generalized Prochhammer's wave propagation theory should be systematically performed in the data processing of wave shift $[21,22]$. In practice, two $3 \mathrm{~m}$ long Nylon bars of 60 $\mathrm{mm}$ of diameter (density $1200 \mathrm{~kg} / \mathrm{m}^{3}$ and speed $1800 \mathrm{~m} / \mathrm{s}$ ) are used.

\subsection{High speed photography}

It is clear that the experimental setups, both standard material testing system and Hopkinson bar, allow only the measurement of the forces and displacement at the interfaces between the platens or the pressure bars and the specimen. Such measurements lead only the global description (the force-crush displacement) of the specimen. However, the local crush mechanism is desired, which can't be given by the experimental setup introduced above, then digital photography is applied to give a supplementary measurement.

Canon EOS 50D is used to record the deforming process of the lattice truss structure in quasi-static testing. In Hopkinson bar impact testing, the high-speed camera, Photron Fastcam SA5, is applied. A MCDL box is added to synchronize the Hopkinson bars results (force-crush displacement curve) with the images.

\section{Experimental results}

\subsection{Overall experimental response}

The specimen is loaded at the rate of $0.025 \mathrm{~mm} / \mathrm{s}$ and about $12 \mathrm{~m} / \mathrm{s}$, which ensure the nominal strain rates (loading rate divided by the height of the core) are $2 \times 10^{-3} \mathrm{~s}^{-1}$ and $10^{3} \mathrm{~s}^{-1}$ respectively.

With the Eq. (1) and Eq. (2), the force/crush displacement curve under impact loading is obtained. The comparison with the quasi-static one is shown in figure 3. Both curves show a common property of lattice core structures, that is, the reaction of the structure is divided into three stages: before buckling, post-buckling and densification. In the first stage, the force increases as the crush displacement increases until the buckling happens, where the force reaches to the peak point. After the buckling happens, the force decreases rapidly. These two stages manifest the structural instability of beam network. The densification stage is commonly observed in many cellular materials, like honeycomb and metal foams. The unique feature of lattice truss core structure is the stair-like increase. This is because of the different contact time instants between the bended beam and the facesheets.

In figure 3 , one interesting observation is that the peak force increase $20 \%$ from quasi-static loading to impact loading. While, as shown in figure 2, the base material doesn't exhibit as much strain rate sensibility as the truss structure. It means that some other source contributes to the rate sensibility of the structure. One possible source is the lateral inertia effect.

As indicated by Calladine and English [23] in 1984 and also by $\mathrm{Su}$ and $\mathrm{Yu}$ [24] in 1995, lateral inertia plays

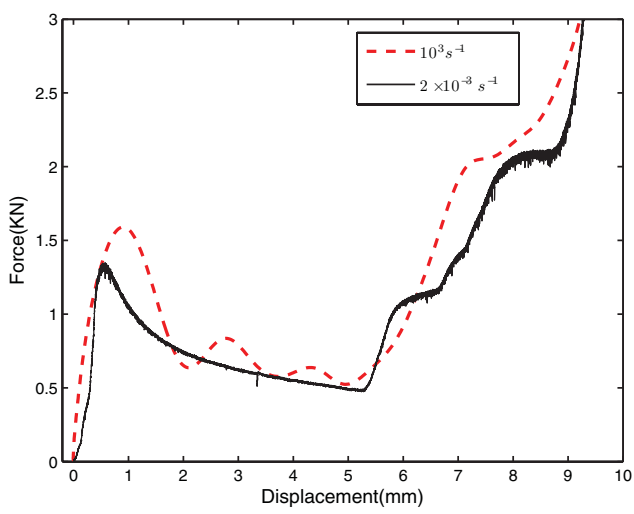

Fig. 3. Comparison of the force/crush displacement under quasistatic loading and impact loading.

a dominant role in the impact problems. Under quasi-static loading, the deformation of the structure predominated by the rotation of plastic hinges. If it's the same in dynamic loading, large transverse speed must reach very soon after the instant of impact to make geometrical compatibility between the structure and the loading end. However, this is not admissible to reach a certain speed in a sudden because it disobeys the laws of mechanics. The mass in the buckling point needs time to be accelerated to a speed which matches the loading speed. Thorefore, the buckling of the beam doesn't occur and the beam keeps relatively straight until the mass in the center is accelerated. This is so-called lateral inertia protection. While during the acceleration period, the compression in longitudinal direction continues, Therefore, the compressive strain reached before collapse under impact loading is therefore higher than in the quasistatic case. In cellular materials, lateral inertia effect was proved to be a key factor of their mechanical responses as well. Zhao et al. [25] pointed that the peak force increase in rate insensitive brass tube is caused by the inertia effect associated with the strain hardening of brass. In our case, the base material A13003-O is a rate insensitive but with a high strain hardening capacity. Then the increase of peak force is assumed to be probably the result of lateral inertia. In next section, image analysis will be used to prove this.

Following the theoretical prediction formulae $[4,6]$, the out-of-plane Young's modulus of the tetrahedral core structure sandwich panel $\left(E_{\text {core }}\right)$ is proportional to relative density $(\bar{\rho})$ of the core:

$$
E_{\text {core }}=E_{s} \sin ^{4} \omega \bar{\rho}
$$

where $E_{s}$ is the Young's modulus of the base material, and $\omega$ is the incline angle plotted in figure 1. It's not clear of the Young's modulus during loading. To observe the variation Young's modulus, the unloading process is executed manually in the displacement increasing about 1 $\mathrm{mm}$. The unloading rate is set to the same value with loading, consequently, the nominal strain rate is the same with loading, $2 \times 10^{-3} \mathrm{~s}^{-1}$. From the unloading and reloading traces, Young's modulus are calculated by averaging the unloading slope and reloading slope. The red points and the red axises in figure 4 indicate the values of Young's modulus at the corresponding displacement and force. A close examination of figure 4 reveals that the Young's 


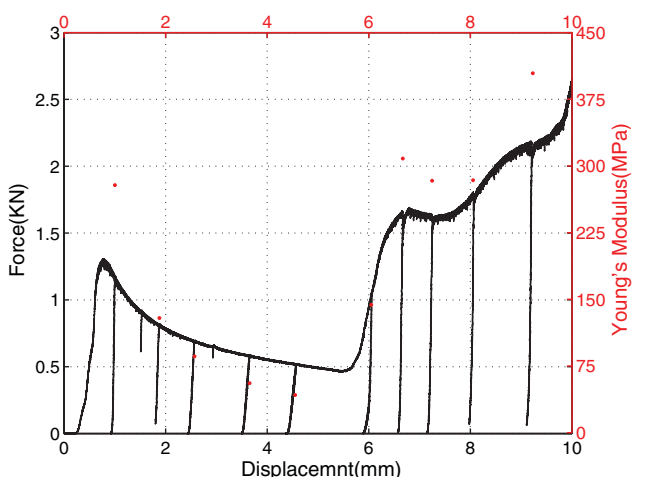

Fig. 4. The quasi-static loading-unloading process: the black curve and the left Y-axis indicate the load; the red points and the right Y-axis indicate the value of Young's modulus.

Modulus were proportional to the forces when unloading occurred.

\subsection{Digital images analysis}

During quasi-static loading, Canon EOS 50D is used in the acquisition frequency $0.33 \mathrm{fps}$ (one image in 3 second). During impact loading, high speed camera Photron SA5 is used to shoot the images of $384 \times 160$ pixels at $100000 \mathrm{fps}$. The use of high speed camera should require much attention to take the images clearly. In order to make the images not blur, the shutter speed is set to very high value, here, $1 / 151000 \mathrm{~s}$ is used. Two high powerful cold-light spotlights are used to provide enough light in high shooting speed and high shutter speed.

By synchronization, the images of the specimen during both quasi-static and dynamic loading are shown figure 5. It compares the deforming states of only one core in the 6 cores specimen at nearly the same crush displacements. The nominal strains labeled on the right of the images are approximations, because it's impossible to find the images from both quasi-static and dynamic loading with the exact crush displacement. From this figure, it's difficult to find whether there is a difference in the deforming mode, because the beams exhibit the same shapes in each crush displacement. What has to be added is that, in repetitive experiments, the two beams in the same plane (the left two beams in each images in figure 5) bend exactly the same, away from or near to the other single beam in the same core. There are four possible combinations of the bending direction in one core (two possible directions of the two beams in the same plane multiply the two possibilities of the other single beam), and in fact the force/crush curves have no obvious differences in no matter which combination.

Even though we can't find obvious differences in different loading cases, the peak force enhancement indeed exists. Meanwhile, the lateral inertia effect introduced above can't not influence too much the deforming state of beam. However, the direct result of inertia effect is larger shortening of the beam, that is the strain on the beam. With the development of digital image correlation method, the full field measurement of strain on the surface of material tested becomes realizable. Unfortunately, in the
Quasi-static
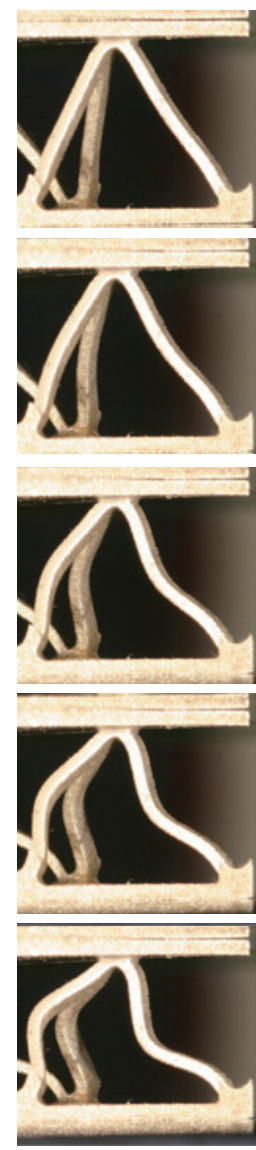

Dynamic
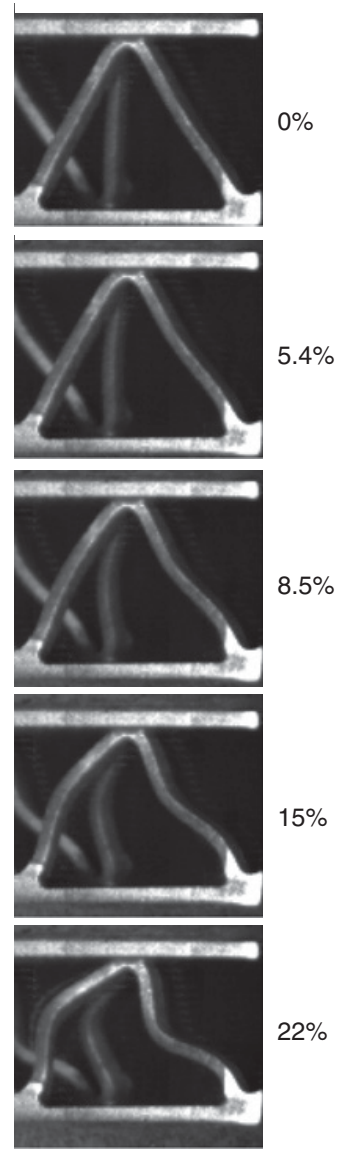

Fig. 5. Comparison of deforming states between quasi-static and dynamic loading at different nominal strain.

high shooting speed during impact loading, the resolution of image is limited. Due to the small thickness of the beam, only 7 pixels are located on the beam. It's not enough to get reliable result by DIC method.

To investigate the details in the images, a digital image analysis method based on the canny edge detection algorithm [26] is developed. We apply a series of small windows, the union of which covers the whole beam concerned and the distances of the centers of windows are less than two pixels in both horizontal and vertical directions. In practice, the small windows with $9 \times 9$ pixels for the images in dynamic loading and $17 \times 17$ pixels for the images in quasi-static loading are adopted. Then the edge detection algorithm in MATLAB is applied directly to detect only one side of the beam (each beam has two sides) in one window. Then the 0-1 matrix, where 1 indicates the edge based on pixel is given by MATLAB. Assuming that the beam is straight, we average the 1-location in $0-1$ matrix in both horizontal and vertical direction to get the sub-pixel accurate edge point of one window. Connect the edge points in adjacent windows pre-generated, the edge of the whole beam is obtained, which is assumed to represent deforming shape of the beam. Apply these processes introduced above into the sequences of images, and then the deforming profile of the beam is extracted. Figure 6 shows the deforming profile under dynamic loading (Only every 5 th traces are plotted to make the figure clear). Thus, the 


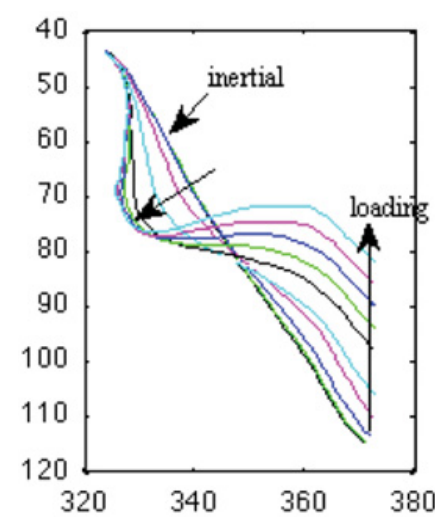

Fig. 6. The extraction of beam deforming profile during impact test.

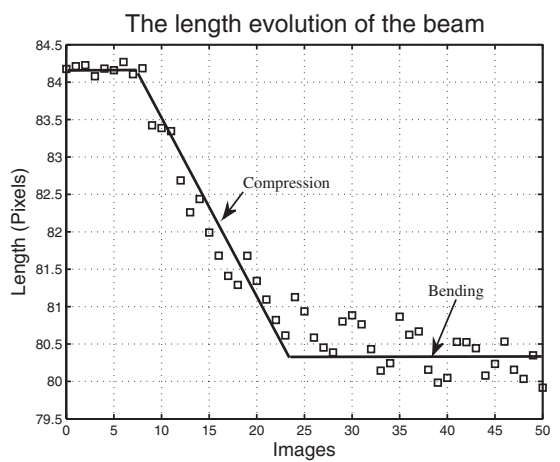

Fig. 7. Beam length evolutions during impact test.

length of beam can be calculated with the knowledge of the deforming profile, see in figure 7 . It is found that the length reduces rather linearly at the early stage and stay nearly constant afterward. Such a measurement can be interpreted as the compression of the beam is rather constant at early stage of loading before the bending becomes dominant.

It's clear that the image shooting angle will influence the shapes of beam in the image and also the absolute lengths of beam exposed to observer, and also due to the difficulty in manipulating the exact same shooting angle in different tests, the absolute lengths is not comparable. Therefore a non-unit value, strain in axial direction, which is defined as the current length divided by original length of beam, is introduced to compare between quasi-static loading and dynamic loading. When the crush arrives 1.2 $\mathrm{mm}$, the quasi-static axial strain is $1.4 \%$ while the impact one is $3.4 \%$, shown in figure 8 . The more compression under dynamic loading proves the existence of lateral inertia effect. With the increase in the axial strain in the beam, the strength of base material increases 30\%, which cause the increase of the peak force shown in figure 3. Considering that the different imperfections of the beams in the specimen will lead different buckling instant, thereupon, decrease the initial peak force, the $20 \%$ strength increase of the specimen but $30 \%$ increase of the base material is relatively acceptable.

Now, with the knowledge of more compressed of beam under impact loading from the image analysis, we conclude that the strength increase of the truss core structure during dynamic loading is caused by the combina-

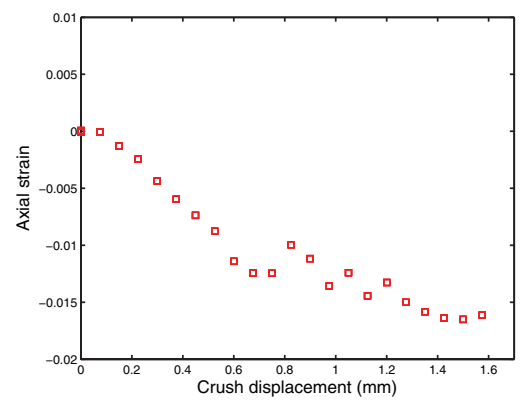

(a) Static

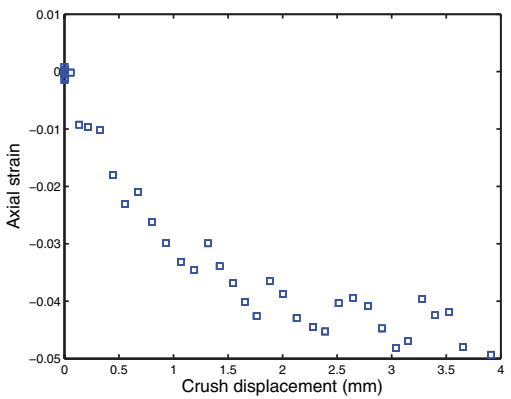

(b) Dynamic

Fig. 8. The axial strain evolutions in quasi-static loading and impact loading.

tion of the lateral inertia effect of the structures and the strong strain hardening capability of Al3003-O (the base material).

\section{Summary}

The out-of-plane compressive behavior of lattice truss core structure was investigated under the nominal strain rate about $2 \times 10^{-1} \mathrm{~s}^{-1}$ and $10^{3} \mathrm{~s}^{-1}$. The increase of initial peak force was observed by comparing the force/crush curve, which is supposed to be dominated by the lateral inertia effect. Digital cameras were applied to record the real time deformation of the specimen. With the digital images analysis, the deforming profile of the beam was extracted. Furthermore, the introduction of a non-unit value, axial strain, gives a credible comparison between different loading cases. It was found that the beam was more compressed under the impact loading, which is an evidence of the existence of lateral inertia.

\section{References}

1. L.J. Gibson, M.F. Ashby, Cellular Solids: Structure and Properties, 2ed (Cambridge University Press, Cambridge, 1997)

2. D.J. Sypeck, Appl. Compos. Mater. 12, (2005) 229246

3. D.J. Sypeck, Metall. Trans. B 36, (2005) 125-131

4. V.S. Deshpande, N.A. Fleck, Int. J. Solids Struct. 38, (2001) 6275-6305

5. D.T. Queheillalt, H.N.G. Wadley, Acta Mater. 53, (2005) 303-313 
6. H.N.G. Wadley, Phil. Trans. R. Soc. A 364, (2005) 3168

7. F.W. Zok, S.A. Waltner, Z. Wei, H.J. Rathbun, R.M. McMeeking, A.G. Evens, Int. J. Solids Struct. 41, (2004) 6249-6271

8. J. Hutchinson, Z. Xue, Int. J. Mech. Sci. 47, (2005) 545-569

9. N.A. Fleck, V.S. Deshpande, J. Appl. Mech. 71, (2004) 386-401

10. K.P. Dharmasena, D.T. Queheillalt, H.N.G. Wadley, P. Dudt, Y. Chen, D. Knight, A. G. Evans, Eur. J. Mech. A. Solids 29, (2010) 56-67

11. X.D. Cui, L. M. Zhao, Z.H. Wang, H. Zhao, D.N. Fang, Int. J. Impact Eng, 43, (2012) 1-5

12. C.J. Yungwirth, H.N.G. Wadley, J.H. O'Connor, A.J. Zakraysek, V.S. Deshpande, Int. J. Impact Eng. 35, (2008) 920-936

13. S. Lee, F. Barthelat, J.W. Hutchinson, H.D. Espinosa, Int. J. Plast. 22, (2006) 2118-2145

14. Exp. Mech. 46, (2006) 789-803

15. J.D. Rehkopf, G.M. Mcneice, G.W. Borland, J. Eng. Mater. Technol. 118, (1996) 58-62
16. F.S. Chang, Y. Song, D.X. Lu, C.N. Desilva, J. Eng. Mater. Technol. 120, (1998) 212-217

17. H.L. Schreyer, Q.H. Zuo, A.K. Maji, J. Eng. Mech. 120, (1994) 1913-1930

18. O. Faruque, N. Liu, C. Chou, SAE Technical Paper 971076 (1997)

19. B. Hopkinson, Philos. Trans. Of the Royal Soc. Of London 213, (1914) 437-456

20. H. Kolsky, Proc. Phys. Soc. B 62, (1949) 676700

21. R.M. Davies, Philos. Trans. Of the Royal Soc. Of London. B 240, (1948) 375-457

22. H. Zhao, G. Gary, J. Mech. Phys. Solids. 43, (1995) 1335-1348

23. C.R. Calladine, R.W. English, Int. J. Mech. Sci. 26, (1984) 689-701

24. X.Y. Su, T.X. Yu, S.R. Reid, Int. J. Impact Eng. 16, (1995) 651-672

25. H. Zhao, S. Abdennadher, Int. J. Solids Struct. 41, (1994) 6677-6697

26. J. Canny, IEEE Trans. Pattern Anal. Mach. Intell. 8, (1986) 679-698 